\section{Review Article}

Check for updates

\section{OPEN ACCESS}

Received: Jun 8, 2019

Corresponding Author:

Won Suk Choi, MD, PhD

Division of Infectious Diseases, Department of Internal Medicine, Korea University College of Medicine, Korea University Ansan Hospital, 123, Jeokgeum-ro, Danwon-gu, Ansan 15355, Gyeonggi-do, Korea.

Tel: +82-31-412-4271

Fax: +82-37-412 5984

E-mail: cmcws@korea.ac.kr

Copyright (C) 2019 by The Korean Society of Infectious Diseases and Korean Society for Antimicrobial Therapy

This is an Open Access article distributed under the terms of the Creative Commons Attribution Non-Commercial License (https:// creativecommons.org/licenses/by-nc/4.0/) which permits unrestricted non-commercial use, distribution, and reproduction in any medium, provided the original work is properly cited.

ORCID iDs

Won Suk Choi (D)

https://orcid.org/0000-0001-5874-4764

Conflict of Interest

No conflicts of interest.

\title{
The National Influenza Surveillance System of Korea
}

\section{Won Suk Choi}

Division of Infectious Diseases, Department of Internal Medicine, Korea University College of Medicine, Seoul, Korea

\section{ABSTRACT}

Influenza is a very important respiratory infectious disease that causes seasonal epidemics and pandemics. A well-organized surveillance system is necessary to monitor and respond effectively to the epidemiologic features of influenza. Korea currently operates a national influenza surveillance system based on the clinical sentinel surveillance system, laboratory sentinel surveillance system, and hospitalization and mortality surveillance system. However, there is a need for a better national surveillance system due to a demand for various pieces of information related to influenza. This article discusses the general aspects of influenza surveillance systems and the future direction of the national influenza surveillance system of Korea.

Keywords: Influenza; Human; Sentinel surveillance; Population surveillance; Korea

\section{INTRODUCTION}

Influenza is a very important respiratory infectious disease that causes seasonal epidemics globally and annually among people of all age groups. Influenza not only causes seasonal epidemics but also causes pandemics every 10 to 40 years. Thus, influenza is considered as one of the most important among various infectious diseases [1]. Although vaccines and antiviral agents can be used in the control of influenza, so far, they have not yielded very satisfactory results $[2,3]$. Therefore, a well-organized surveillance system is necessary to monitor and respond effectively to influenza epidemics.

An influenza surveillance system is useful for identifying influenza epidemics, the nature of the virus, and the burden of disease, as well as determining the policies aimed at influenza epidemic control, vaccine selection, and the development of guidelines for the use of antiviral drugs. An influenza surveillance system can also be used to monitor influenza pandemics and new respiratory infectious diseases. Korea currently operates a national influenza surveillance system based on the clinical sentinel surveillance system, laboratory sentinel surveillance system, and hospitalization and mortality surveillance system. However, there is a need for a better national surveillance system due to the increased demand for various pieces of information related to influenza. This article reviews the influenza surveillance system characteristics, foreign surveillance systems, including those of the World Health 
Organization (WHO) and the United States (US), and the influenza surveillance system of Korea. It further discusses the future direction of the national influenza surveillance system of Korea.

\section{INFLUENZA SURVEILLANCE SYSTEMS IN GENERAL}

Infectious disease surveillance systems can be classified into general or sentinel, clinical or laboratory-based, or passive or active surveillance systems [4]. In countries operating influenza surveillance systems, the most common form is active sentinel surveillance, which involves the use of both clinical and laboratory data [5-8].

There are two major forms of influenza surveillance systems: clinical sentinel surveillance and virologic sentinel surveillance systems [9]. A clinical sentinel surveillance system is used to identify influenza outbreaks and epidemics, establish a vaccination plan, initiate antiviral agent use, and measure the burden of influenza, which are the basic data required for public health policies for influenza. The most important indicators in clinical sentinel surveillance are the influenza-like illness (ILI) incidence and influenza-associated mortality. In addition, other indicators can be used that represent the burden of influenza in the community such as absenteeism, or that show the severity of influenza or burden from the healthcare perspective such as emergency department visit ratio and hospitalization ratio. Virologic sentinel surveillance is used to identify the characteristics of circulating viruses, which include resistance to antiviral agents, and matching of vaccine strains and circulating strains. Since clinical sentinel surveillance and virologic sentinel surveillance play a complementary role, they should not be operated separately; they should ideally be operated concurrently by selecting representative sentinel sites.

Clinical sentinel surveillance systems are classified into the outpatient department (OPD)based surveillance and hospitalization-based surveillance systems. OPD-based surveillance systems are used to determine the occurrence of influenza epidemics by monitoring the ratio of the number of ILI patients relative to all outpatients. The sites assessed during OPD-based surveillance should be selected based on their geographical distribution and population characteristics among OPD clinics that directly treat influenza patients. The selected sites report the information of patients with ILI predefined on a daily or weekly basis via internet, telephone or fax. The collected data are interpreted taking into consideration the characteristics of the participating sites, virologic surveillance data, geographical and demographic characteristics, and influenza vaccination rate. The hospitalization-based surveillance system contributes to the understanding of the severity and burden of influenza by monitoring cases of severe acute respiratory illness (SARI), hospitalization, intensive care unit (ICU) care, and mortality. Influenza is more likely to cause complications or severe progression than other respiratory viruses. The main anticipated effect of influenza vaccination is to reduce the burden of influenza. Therefore, a hospitalization-based surveillance system is essential to determine the appropriateness and evaluate the effectiveness of policies for influenza control. Data collected through both surveillance systems can be used to assess influenza vaccine effectiveness. However, a procedure for increasing the accuracy of influenza vaccination history is required to evaluate the influenza vaccine effectiveness accurately. In addition, a sufficient number of patients should be included in the surveillance to assess the vaccine effect by virus type/subtypes and age groups. 


\section{FOREIGN INFLUENZA SURVEILLANCE SYSTEMS}

\section{World Health Organization}

The WHO discussion on the operation of the influenza network began in 1951 at a conference held in Copenhagen, and in 1952, the WHO Global Influenza Surveillance Network was launched [10]. In May 2011, the Pandemic Influenza Preparedness Framework was adopted, which involved sharing of influenza virus characteristics and other benefits of vaccines, and its name was changed to Global Influenza Surveillance and Response System (GISRS). The GISRS consists of 144 national influenza centers, 6 WHO collaborating centers (CCs), 4 essential regulatory laboratories, and $13 \mathrm{H} 5$ reference laboratories (H5RefLabs) as of 2019 [11].

National influenza centers collect virus specimens, and ship representative clinical specimens and isolated viruses to WHO CCs for advanced antigenic and genetic analysis. The WHO recommends the composition of influenza vaccines based on the results of the analysis. WHO CCs are responsible for the development of literature and research. One of the six WHO CCs is responsible for surveillance, epidemiology, and influenza prevention. Essential regulatory laboratories have the critical function of connecting surveillance and risk assessment activities with influenza vaccine response. H5RefLabs were established in 2004 , in response to the public health needs arising from avian influenza A/H5N1 infection in humans and influenza pandemic preparedness. H5RefLabs collect and analyze influenza $\mathrm{A} / \mathrm{H} 5 \mathrm{~N} 1$ or other influenza subtypes that can potentially cause human infections. The data collected by each institution of the GISRS are uploaded to the WHO database and presented via FluNet, a global web-based tool for influenza virologic surveillance first launched in 1997. The virologic data presented via FluNet are crucial to tracking the movement of viruses and interpreting the epidemiological data. The WHO also operates a data sharing platform called FluID, which provides connections between existing databases.

Currently, the WHO recommends evaluating disease burden using surveillance data from each country [12]. Thus, in many countries, data on the burden of influenza are being prepared [13-16].

\section{United States}

The US Centers for Disease Control and Prevention (CDC) collect five categories of information from eight different data sources on influenza activity year-round: virologic surveillance, outpatient illness surveillance, summary of the geographic spread of influenza, hospitalization surveillance, and mortality surveillance [17]. The analysis results are presented through FluView and FluView Interactive.

Virologic surveillance. Approximately 100 public health laboratories and over 300 clinical laboratories participate in the virologic surveillance: the US WHO Collaborating Laboratories System and the National Respiratory and Enteric Virus Surveillance System. The clinical laboratories provide data on the weekly total number of specimens tested, the number of positive influenza test results, and the percentage of positive test results by influenza virus type. Public health laboratories also report other kinds of data on the weekly total number of specimens tested, the number of positive influenza test results, and the number by influenza virus type, subtype, and influenza B lineage.

Outpatient illness surveillance. Information on outpatient visits to health care providers for ILI is collected through the US Outpatient Influenza-like Illness Surveillance Network 
(ILINet). ILINet consists of more than 3,500 enrolled outpatient healthcare providers. They report data on the total number of patients seen for any reason and the number of those patients with ILI by age group ( $0-4$ years, $5-24$ years, $25-49$ years, $50-64$ years, and $\geq 65$ years). ILI is defined as fever (temperature of $37.8^{\circ} \mathrm{C}$ or greater) and a cough and/or sore throat without a known cause other than influenza. Based on the ILINet results, state health departments report the estimated level of the geographic spread of influenza activity in their states each week through the State and Territorial Epidemiologists Reports. The influenza activity is classified at five levels: no activity, sporadic, local, regional, or widespread.

Summary of the Geographic Spread of Influenza. Each state reports on the weekly geographic spread of influenza activity through the State and Territorial Epidemiologists Reports. Influenza activity is reported as no activity, sporadic, local, regional, and widespread.

Hospitalization surveillance. The Influenza Hospitalization Surveillance Network monitors laboratory-confirmed influenza-associated hospitalization. During the influenza season, data are collected from cases with laboratory-confirmed influenza. Based on the surveillance data, age-specific hospitalization rates and patients' characteristics are presented through FluView Interactive weekly.

Mortality surveillance. Influenza-associated mortality surveillance consists of two systems. The first is the National Center for Health Statistics mortality surveillance system. The National Center for Health Statistics collects pneumonia and influenza mortality data from each state based on ICD-10 codes. The seasonal baseline pneumonia and influenza mortality rate is estimated using a periodic regression model. The epidemic threshold is estimated by an increase of 1.645 standard deviations above the baseline. The second is the InfluenzaAssociated Pediatric Mortality Surveillance System. Since 2004, deaths among children younger than 18 years with laboratory-confirmed influenza have been added as a nationally notifiable condition.

In addition, the US has been operating a vaccine effectiveness surveillance [18]. Surveillance for monitoring influenza vaccine effectiveness is managed separately by the US Flu VE Network, which focuses on outpatients, and the US Hospitalized Influenza Vaccine Effectiveness Network, which focuses on inpatients. Each network consists of five sites in the US. At the participating sites, respiratory specimens are collected for isolating influenza viruses together with demographic and medical information, including underlying diseases and influenza vaccination history. The two networks assess and report influenza vaccine effectiveness separately during each season $[19,20]$. The US CDC calculates and reports the influenza disease burden using the Influenza surveillance data, and the averted burden by vaccine based on the surveillance and estimated data [21].

\section{Europe}

The European Centre for Disease Prevention and Control (ECDC) coordinates the Influenza Surveillance Network (EISN) [22]. The aim of the EISN is to contribute to reducing the influenza burden in Europe. The EISN originated in the European Influenza Surveillance Scheme (EISS) by the Netherlands Institute for Health Services Research. The EISS started in 1996 and was Europe's initial surveillance network. Its name was changed to EISN in September 2008 and is currently operated by the ECDC. The EISN has 50 member states in Europe. Each member state has a separate influenza surveillance system and case definition 
for the surveillance. The form of surveillance also varies depending on the region. Each member state reports epidemiologic and virologic data to The European Surveillance System, which is hosted by the ECDC. Surveillance is mainly based on nationally organized sentinel networks of primary care physicians, which cover at least 1-5\% of the population of each country. Virologic surveillance activities are coordinated through the European Reference Laboratory Network for Human Influenza, a sub-network of the EISN. For the surveillance of severe influenza, 16 member states conduct sentinel surveillance for hospitalized cases with SARI, and 13 member states report laboratory-confirmed influenza-positive cases from intensive care units.

\section{Japan}

The national influenza surveillance system of Japan is affiliated to the National Epidemiological Surveillance for Infectious Diseases (NESID), which includes a mandatory reporting system for nationally notifiable diseases and sentinel surveillance systems for various kinds of infectious diseases including influenza [23]. The sentinel outpatient surveillance system has been applied to influenza surveillance. Sentinel sites were designated according to the geographic distribution, type of medical institutions, and population densities. Sentinel sites report the age group and sex of patients with ILI every week. Virologic surveillance, consisting of testing for influenza virus and the analysis of viral antigenicity, is incorporated into outpatient surveillance under the scheme of NESID. About 500 medical institutions out of 5,000 sentinel sites are designated as pathogen collection sites. In addition to the outpatient and virologic surveillance, data on the number of influenza-associated hospitalizations are also collected under NESID. Around 500 hospitals with more than 300 beds are selected as sentinel sites for hospitalization surveillance. Japan has monitored the incidence of influenza-associated encephalopathy since the late 1990s. Japan has a surveillance system for student absence and school closures. The law of Japan states that children with influenza infection should stay home for at least 6 days after the onset of symptoms. The act also designates that the school can be closed to stop the transmission of influenza depending on the size of influenza outbreaks. In addition to the surveillance system operated by the public sector, there is a volunteer-based web ILI surveillance system. The surveillance system is organized by volunteer clinicians who report the number of influenza patients diagnosed at their clinics through the ML-Influenza Database.

\section{THE CURRENT INFLUENZA SURVEILLANCE SYSTEM OF KOREA}

The influenza surveillance system of Korea was initiated in October 1997 with a small number of volunteer specialists in internal medicine, pediatrics, and family medicine [9]. This surveillance system has been extended since 2000 to the Korea Influenza Surveillance Scheme. Currently, the influenza surveillance system operated by the Korea CDC consists of clinical sentinel surveillance, laboratory sentinel surveillance (Korea Influenza and Respiratory Virus Surveillance System [KINRESS]), and influenza hospitalization and mortality surveillance systems [24].

Clinical sentinel surveillance. The clinical sentinel surveillance is operated by selecting 200 outpatient clinics with the recommendation of a medical association. It is required to report the number of ILI patients and the total number of outpatients by age groups. Reports are provided every week from April to November and daily from December to April of the following year. The data are used to identify influenza epidemics in Korea. 
Laboratory sentinel surveillance. KINRESS is operated at 52 sites (as of July 2018) among the clinics participating in the clinical sentinel surveillance. The sites participating in KINRESS collect respiratory specimens from patients with ILI and send them to the regional Research Institutes of Public Health and Environment. The institutes conduct gene detection tests and send the results and residual samples to the KCDC. The KCDC conducts virus identification and reexamination of unidentified specimens. Based on these results, the circulating influenza viruses in Korea are identified, and the characteristics of the viruses are revealed, including resistance to antiviral agents and matched with the vaccine strain.

Influenza hospitalization and mortality surveillance. Influenza hospitalization and mortality surveillance are being conducted at 207 hospitals (as of July 2018) participating in Acute Respiratory Infection Sentinel Surveillance in Korea. The hospitals participating in the surveillance report statistics on new inpatients, outpatients, and emergency room patients diagnosed with influenza, as well as the number of deaths among patients diagnosed with influenza.

The Hospital-based Influenza Morbidity and Mortality (HIMM) surveillance system has been operated since 2011 as a research project of the Transgovernmental Enterprise for Pandemic Influenza in Korea [25]. The HIMM system is a kind of SARI surveillance system and has ten participating university hospitals. Respiratory specimens are collected at the sites for patients who visited the ER with ILI or hospitalized patients diagnosed with influenza. Medical histories are also collected, including influenza vaccination history, underlying medical condition, travel history, and the progress of influenza. Influenzarelated statistics and collected data are reported on a daily and weekly basis. The HIMM data have been used to analyze the clinical course of severe influenza [26, 27], influenza disease burden [28], characteristics of influenza viruses that caused severe clinical course [29], influenza vaccine effectiveness, and so on. In addition to surveillance for severe influenza, there is surveillance for avian influenza viruses that can cause human infection such as $\mathrm{A} / \mathrm{H} 7 \mathrm{~N} 9$ and $\mathrm{A} / \mathrm{H} 5 \mathrm{~N} 1$ [30]. Currently, the HIMM surveillance system continues to be operated at nine hospitals.

\section{THE FUTURE DIRECTION OF INFLUENZA SURVEILLANCE SYSTEMS IN KOREA}

Influenza comprises not only a burden on the medical system but also has a huge socioeconomic burden in Korea. A large part of the government budget has been used to target influenza vaccination. Influenza vaccination among the elderly has been included in the national immunization program since 1997, and children have been included since 2015 [31]. Influenza vaccination for pregnant women will also be included in the national immunization program from the 2019-2020 season. Considering the impact of influenza and the continuous change in the characteristics of influenza viruses, a proper surveillance system is essential in Korea. The national influenza surveillance system currently operated by the government has played an important role in identifying influenza epidemic patterns and virus characteristics in Korea. However, it has limitations and needs to be supplemented.

First, the current surveillance system operated by the KCDC provides a limited understanding of the characteristics of serious influenza and its disease burden. The hospitalization and mortality surveillance system is being operated, but the data collected are very limited 
and cannot be used to fully understand the clinical course. It is also difficult to identify the characteristics of viruses associated with severe influenza because specimens are not collected. If it is not possible to collect enough data on severe influenza, it would be difficult to fully assess the burden of influenza. In the US, when assessing the burden of influenza illness, the spectrum of influenza from mild to dead is estimated based on hospitalized patients. Another limitation is the difficulty in assessing the effectiveness of influenza vaccines. Although information regarding influenza vaccination history has been collected, the reliability of the information is insufficient. In order to ensure a reliable vaccination history, it is necessary to approach history taking via various methods such as reviewing past medical records, reviewing vaccine registration data, and in-depth interview. However, it is difficult to obtain such reliable data in the current surveillance system. In addition, evaluating vaccine effectiveness among outpatients would yield unrepresentative results, so data should be collected from both outpatients and inpatients. An example is the surveillance system for influenza vaccine effectiveness in the US. The assessment of the burden of influenza and the evaluation of the vaccine effectiveness is very important not only in the determination of policy direction and assessment of the impact, but also in the risk-communication with people on the influenza. The surveillance system should have a clear objective, and a single system cannot fulfill every purpose. To meet the unmet need of influenza surveillance in Korea, therefore, it does not simply suffice to adjust the data collected from the existing influenza surveillance system.

The national influenza surveillance system currently operated in Korea is relatively wellorganized and has contributed to our understanding of the characteristics of influenza. However, more needs to be done to better cope with influenza considering the influenza situation of Korea and foreign surveillance systems. A better national surveillance system will support proper policy making and produce enough scientific evidence regarding influenza control.

\section{REFERENCES}

1. Cassini A, Colzani E, Pini A, Mangen MJ, Plass D, McDonald SA, Maringhini G, van Lier A, Haagsma JA, Havelaar AH, Kramarz P, Kretzschmar ME. On Behalf Of The BCoDE Consortium. Impact of infectious diseases on population health using incidence-based disability-adjusted life years (DALYs): results from the Burden of Communicable Diseases in Europe study, European Union and European Economic Area countries, 2009 to 2013. Euro Surveill 2018;23:17-00454. CROSSREF

2. Gross PA, Hermogenes AW, Sacks HS, Lau J, Levandowski RA. The efficacy of influenza vaccine in elderly persons. A meta-analysis and review of the literature. Ann Intern Med 1995;123:518-27. PUBMED | CROSSREF

3. Doll MK, Winters N, Boikos C, Kraicer-Melamed H, Gore G, Quach C. Safety and effectiveness of neuraminidase inhibitors for influenza treatment, prophylaxis, and outbreak control: a systematic review of systematic reviews and/or meta-analyses. J Antimicrob Chemother 2017;72:2990-3007. PUBMED | CROSSREF

4. Park O, Choi BY. Introduction and evaluation of communicable disease surveillance in the republic of Korea. J Prev Med Public Health 2007;40:259-64. PUBMED | CROSSREF

5. Sanicas M, Forleo E, Pozzi G, Diop D. A review of the surveillance systems of influenza in selected countries in the tropical region. Pan Afr Med J 2014;19:121. PUBMED | CROSSREF

6. Thommes EW, Kruse M, Kohli M, Sharma R, Noorduyn SG. Review of seasonal influenza in Canada: Burden of disease and the cost-effectiveness of quadrivalent inactivated influenza vaccines. Hum Vaccin Immunother 2017;13:867-76. PUBMED | CROSSREF 
7. Sambala EZ, Ndwandwe DE, Imaan LM, Wiysonge CS. Evaluation of influenza surveillance systems in sub-Saharan Africa: a systematic review protocol. BMJ Open 2019;9:e023335. PUBMED | CROSSREF

8. Yang P, Duan W, Lv M, Shi W, Peng X, Wang X, Lu Y, Liang H, Seale H, Pang X, Wang Q. Review of an influenza surveillance system, Beijing, People's Republic of China. Emerg Infect Dis 2009;15:1603-8. PUBMED | CROSSREF

9. Kim WJ, Noh JY, Park MS, Song JY, Shin O, Ahn DH, Wie SH, Lee JS, Jang J, Cheong HJ, Chun BC, Choi YK, Choi WS. Influenza. Seoul: Transgovernmental Enterprise for Pandemic Influenza in Korea; 2016.

10. Ziegler T, Mamahit A, Cox NJ. 65 years of influenza surveillance by a World Health Organizationcoordinated global network. Influenza Other Respir Viruses 2018;12:558-65. PUBMED | CROSSREF

11. World Heaith Organization. Global influenza surveillance and response system (GISRS). Available at: https://www.who.int/influenza/gisrs_laboratory/en/. Accessed 22 April 2019.

12. Chaiyakunapruk N, Kotirum S, Newall AT, Lambach P, Hutubessy RCW. Rationale and opportunities in estimating the economic burden of seasonal influenza across countries using a standardized WHO tool and manual. Influenza Other Respir Viruses 2018;12:13-21. PUBMED | CROSSREF

13. Oliva J, Delgado-Sanz C, Larrauri A; Spanish Influenza Surveillance System. Estimating the burden of seasonal influenza in Spain from surveillance of mild and severe influenza disease, 2010-2016. Influenza Other Respir Viruses 2018;12:161-70. PUBMED | CROSSREF

14. Schanzer DL, Saboui M, Lee L, Nwosu A, Bancej C. Burden of influenza, respiratory syncytial virus, and other respiratory viruses and the completeness of respiratory viral identification among respiratory inpatients, Canada, 2003-2014. Influenza Other Respir Viruses 2018;12:113-21. PUBMED | CROSSREF

15. Simmerman JM, Uyeki TM. The burden of influenza in East and South-East Asia: a review of the English language literature. Influenza Other Respir Viruses 2008;2:81-92. PUBMED | CROSSREF

16. Bresee J, Fitzner J, Campbell H, Cohen C, Cozza V, Jara J, Krishnan A, Lee V, Working WH; WHO Working Group on the Burden of Influenza Disease. Group on the Burden of Influenza Disease. Progress and remaining gaps in estimating the global disease burden of influenza. Emerg Infect Dis 2018;24:1173-7. PUBMED | CROSSREF

17. Centers for Disease Control and Prevention. Overview of influenza surveillance in the United States. Available at: https://www.cdc.gov/flu/weekly/overview.htm. Accessed 22 April 2019.

18. Centers for Disease Control and Prevention. CDC seasonal flu vaccine effectiveness studies. Available at: https://www.cdc.gov/flu/vaccines-work/effectiveness-studies.htm. Accessed 22 April 2019.

19. Treanor JJ, Talbot HK, Ohmit SE, Coleman LA, Thompson MG, Cheng PY, Petrie JG, Lofthus G, Meece JK, Williams JV, Berman L, Breese Hall C, Monto AS, Griffin MR, Belongia E, Shay DK; US Flu-VE Network. Effectiveness of seasonal influenza vaccines in the United States during a season with circulation of all three vaccine strains. Clin Infect Dis 2012;55:951-9. PUBMED | CROSSREF

20. Ferdinands JM, Gaglani M, Martin ET, Middleton D, Monto AS, Murthy K, Silveira FP, Talbot HK, Zimmerman R, Alyanak E, Strickland C, Spencer S, Fry AM; HAIVEN Study Investigators. Prevention of influenza hospitalization among adults in the US, 2015-16: Results from the US Hospitalized Adult Influenza Vaccine Effectiveness Network (HAIVEN). J Infect Dis 2018; ; Epub ahead of print. PUBMED | CROSSREF

21. Rolfes MA, Foppa IM, Garg S, Flannery B, Brammer L, Singleton JA, Burns E, Jernigan D, Olsen SJ, Bresee J, Reed C. Annual estimates of the burden of seasonal influenza in the United States: a tool for strengthening influenza surveillance and preparedness. Influenza Other Respir Viruses 2018;12:132-7. PUBMED | CROSSREF

22. European Centre for Disease Prevention and Control. European influenza surveillance network (EISN). Available at: https://ecdc.europa.eu/en/about-us/partnerships-and-networks/disease-and-laboratorynetworks/eisn. Accessed 2 April 2019.

23. Zaraket H, Saito R. Japanese surveillance systems and treatment for influenza. Curr Treat Options Infect Dis 2016;8:311-28. PUBMED | CROSSREF

24. Korea Centers for Disease Control and Prevention (KCDC). 2018-2019 season influenza management guideline. Osong: KCDC; 2018. 
25. Seo YB, Song JY, Cheong HJ, Cho YD, Wie SH, Jeong HW, Kim WJ. Hospital-based influenza morbidity and mortality surveillance system for influenza-like illnesses: a comparison with national influenza surveillance systems. Influenza Other Respir Viruses 2014;8:17-20. PUBMED | CROSSREF

26. Hong KW, Cheong HJ, Choi WS, Lee J, Wie SH, Baek JH, Kim HY, Jeong HW, Kim WJ. Clinical courses and outcomes of hospitalized adult patients with seasonal influenza in Korea, 2011-2012: Hospital-based Influenza Morbidity \& Mortality (HIMM) surveillance. J Infect Chemother 2014;20:9-14. PUBMED | CROSSREF

27. Kang SH, Cheong HJ, Song JY, Noh JY, Jeon JH, Choi MJ, Lee J, Seo YB, Lee JS, Wie SH, Jeong HW, Kim YK, Park KH, Kim SW, Jeong EJ, Lee SH, Choi WS, Kim WJ. Analysis of risk factors for severe acute respiratory infection and pneumonia and among adult patients with acute respiratory illness during 20112014 influenza seasons in Korea. Infect Chemother 2016;48:294-301. PUBMED | CROSSREF

28. Choi WS, Cowling BJ, Noh JY, Song JY, Wie SH, Lee JS, Seo YB, Lee J, Jeong HW, Kim YK, Kim SW, Park KH, Lee SH, Cheong HJ, Kim WJ. Disease burden of 2013-2014 seasonal influenza in adults in Korea. PLoS One 2017;12:e0172012. PUBMED | CROSSREF

29. Lee HS, Noh JY, Song JY, Cheong HJ, Choi WS, Jeong HW, Wie SH, Kim WJ. Molecular genetic characteristics of influenza A virus clinically isolated during 2011-2016 influenza seasons in Korea. Influenza Other Respir Viruses 2018;12:497-507. PUBMED | CROSSREF

30. Song JY, Noh JY, Lee J, Woo HJ, Lee JS, Wie SH, Kim YK, Jeong HW, Kim SW, Lee SH, Park KH, Kang SH, Kee SY, Kim TH, Choo EJ, Lee HS, Choi WS, Cheong HJ, Kim WJ. Hospital-based influenza morbidity and mortality (HIMM) surveillance for A/H7N9 influenza virus infection in returning travelers. J Korean Med Sci 2018;33:e49. PUBMED | CROSSREF

31. Korea Centers for Disease Control and Prevention (KCDC). National immunization program management guideline for 2017-2018 seonal influenza. Osong: KCDC; 2017. 